\title{
Fusion Miner: Process Discovery for Mixed-Paradigm Models
}

\author{
Johannes De Smedt ${ }^{1}$, Jochen De Weerdt, Jan Vanthienen \\ $K U$ Leuven Faculty of Economics and Business Department of Decision Sciences and \\ Information Management Naamsestraat 69 B-3000 Leuven, Belgium
}

\begin{abstract}
The research area of business process mining has vastly matured in recent years.

Its main focus centers around the extraction and analysis of process models from event logs. A strong emphasis lies on the automatic discovery of models for which numerous algorithms have been proposed already. So far, most discovery algorithms were limited to the derivation of single-paradigm models, which contain either procedural or declarative constructs, targeting the mining of strict and flexible processes respectively. This paper proposes the first fullyautomated mining technique to discover procedural workflows combined with Declare templates to capture processes that are difficult to mine with only a single paradigm, e.g. workflows with different layers of flexibility.

This approach provides process analysts with new discovery capabilities, including the retrieval of better fitting and more precise models with high comprehensibility. The main contribution consists of the Fusion Miner algorithm, which has been implemented in the process mining framework ProM as a plugin.

Keywords: Business Process mining, Workflow models, Declare
\end{abstract}

\footnotetext{
${ }^{1}$ Corresponding author.

E-mail: Johannes.DeSmedt@kuleuven.be
} 


\section{Introduction}

Business practitioners often face the need to gain insights into how their business processes are actually being carried out. They query whether the current enactment of processes such as purchase-to-pay, fault-to-resolution, or invoice5 to-cash, etc. are in line with how the process is envisioned, either described in documents such as process models, but also in terms of expectations. In the case of misalignments, adaptation is needed to ensure that decisions made by business owners are reflected in the information system under scrutiny. The field of process mining $[\mathbb{I}]$, which fits under the umbrella of information mining [2], addresses this challenge by focusing on the automatic retrieval and subsequent analysis of business process models and insights from data logs containing events. As such, process mining can be a powerful approach for decision makers for assessing and improving business practices. It does so according to three pillars, process discovery, enhancement and conformance checking. The former can be considered the primordial task in a process mining exercise, supporting the two latter pillars that typically build on top of it. The goal of process discovery is to learn a process model from data in the form of an event log in the most comprehensive, comprehensible and correct way. In order to do so, many mining algorithms have been proposed, including, amongst others, Alpha Miner [3] and Heuristics Miner [4]. Generally, these miners retrieve procedural models, containing strict sequence information, such as Petri nets [5].

More recently, declarative process modeling and mining has gained popularity and several discovery techniques such as, e.g., Declare Miner [6] have been implemented for discovery purposes. These miners derive rules from event logs to create models with a more flexible view on the information contained in the $\log$, as any behavior that is not strictly forbidden is allowed.

In accordance with the "maps" view on process models proposed in [I], one retrieves different information by mining for different paradigms. Similar to reading maps with different perspectives, which cover multiple layers of an area, 30 it is possible to retrieve different paradigms at once to gain complementary 
insights from the information retrieved from the log. For example, combining street maps with altitude information can provide a deeper understanding of the explored area.

In line with this metaphor, this paper presents an approach based on the principles of the authors' work in [7] for combining procedural model fragments and declarative constraints in one map. As such, a new way of mining and representing process models is proposed. This process mining approach exploits the different characteristics of both process paradigms, with each paradigm better capable of representing distinct behavioral aspects of an event log. In the literature, the combination of both paradigms into one discovery algorithm has received very little attention so far. Nonetheless, the prospect of learning richer, mixed-paradigm process models seems promising, especially in the context of semi- or unstructured processes. Our proposed approach has been implemented in ProM $\mathrm{M}^{\square}$ as a plug-in and borrows some key principles of the two most fre45 quently used process mining techniques for each paradigm, Heuristics Miner [4] and Declare Miner [6]. The results show models that are fitting and precise for event logs that contain different layers of flexibility, meaning they show behavior consisting of both strict event sequences and rather loosely defined event occurrences. This improves current single-paradigm approaches regarding both conformance and comprehensibility, hence supporting the initial problem of discovering, enacting and improving current decision making processes. Furthermore, the proposed approach provides nice visualization to allow decision makers to read and inform themselves quickly and in an elaborate way.

The remainder of this paper is structured as follows. The second section covers the related works, followed by a section that elaborates on the authors' take on mixed-paradigm models and the algorithm to mine and criteria to evaluate them. The fourth section evaluates two simulated examples and one real-life case by comparing current techniques and the outcome of Fusion Miner. The last section concludes the paper with a discussion and future work.

\footnotetext{
${ }^{2}$ http://www.processmining.org/
} 


\section{Related work}

Within the field of process mining, a strong emphasis is put on the automatic retrieval of business process models from event logs. The algorithms offer mining solutions that deal with aspects such as the trade-off between recall, precision, generalization, and the presence of noise in the event $\log [\overline{[}]$. Initially, procedural process mining approaches were put forward. Some well known and widely used ones are [9, 3, 4, [0, Ш]]. They capture sequence constraints and parallelism by incorporating information supporting adjacency and (direct) succession in a process log, extended with (X)OR- and AND-split and -join information. Most of the constraints are locally defined (an exception is Fuzzy Miner, which uses an optional distance metric for deriving sequence constraints), but calculated on log level.

More recently, the modeling and mining of flexible process models has gained popularity among researchers. The most prominent declarative control flow modeling framework is Declare [12], which offers a set of Linear Temporal Logic 75 (LTL)-based constraint templates for modeling and rule verification purposes, bundled in the ConDec language. An overview of the most commonly used templates can be found in Table 6. Many declarative process discovery algorithms have been developed, such as [6, [13, 14, [15]. The technique in [6] mines directly for automata of Declare constraints using Apriori based rule learning [16]. In [13], the authors use distinct support functions for each constraint. The use of automata extracted from regular expressions for mining purposes is proposed in [14]. The last algorithm presented in [15] uses SCIFF [17], a declarative language based on computational logic. While different languages are used, the outcome is still represented or can be represented with Declare.

There exist some approaches on the verge between both paradigms, such as Fuzzy Miner [10], UnconstrainedMiner [14] and AGNEs Miner [II8]. The former captures flexible processes by incorporating multiple perspectives such as social network and control flow information (note that this is different from a mixed-paradigm approach). The algorithm makes it possible to visualize process 
have the highest impact on these perspectives, which enables the capturing of flexible models better than, e.g., Alpha Miner. UnconstrainedMiner approaches the mixed-paradigm perspective the other way around, starting from a declarative constraint mining implementation which can incorporate any constraint procedural constraints. A similar approach is used in [18], where the authors propose a discovery algorithm based on NS (no-sequel), a declarative predicate which can be used to derive constructs that form a Petri net.

The field of business process modeling has various solutions for combining purpose is somewhat similar to the one pursued in this paper, there are some noticeable differences. First of all, the authors assume atomic subprocesses, which are still disjoint. This restricts synergies between both paradigms in terms of state space, as they cannot interact to mine and represent process behavior. 120 declarative, flexible model constructs and procedural languages. Pockets of flexibility [[19], worklets [20], and the combination of YAWL and Declare [12] introduce atomic subworkflows into procedural workflows, hence making certain parts of the model more flexible. However, the execution semantics of both modeling types are still separate, as every paradigm still executes in its own part of the model hierarchy. A truly mixed-paradigm approach, that is one with intertwined state-spaces, is proposed in [2] for Petri nets and Declare. Another approach is the incorporation of rules into procedural models [ [22] by calculating process trees and applying change operations on the tree for data constraints afterwards. The scope of this work, however, is more focused on including data aspects into a process model.

A mixed-paradigm outcome in process mining, however, has not been pursued yet as such, with the very recent exception of [2:3], in which the authors break down the event log into a hierarchy and mine the different subprocesses according to the appropriate paradigm. Note that this is similar to the principles of the mixed-paradigm approaches based on hierarchy cited above. While the Furthermore, while the authors propose an approach based on counting pre- 
decessors and successors which is somewhat comparable to direct succession in Section $[2$, applying a threshold on the exact number of predecessors and successors seems rather coarse for deciding on the structured versus unstructured nature of activities, especially for smaller logs. Therefore, our approach includes

125 a configurable threshold based on the concept of entropy, which allows for making a more versatile trade-off between structured and unstructured behavior. In addition, the mandatory hierarchical structure of the mined hybrid model in [23] puts a limitation on its application to event logs where structured and unstructured behavior are much more intertwined. The approach presented in this paper does not presume such a hierarchical structure. Finally, the approach is not setup as a discovery algorithm and serves rather as a log preprocessor.

\section{Mixed-Paradigm Mining and Fusion Miner}

In this section, a definition of mixed-paradigm models is provided, followed by the Fusion Miner algorithm and the evaluation criteria that can be used for mixed-paradigm models.

\subsection{Mixed-Paradigm Models for Mining}

The models used in this paper are based on dependency nets, which can be converted to Petri nets, and Declare. In this paper we assume to execute these models as described in [21]. The work provides an in-depth analysis of executing Declare constraint automata and Petri nets. The authors suggest three ways of enactment (the last one only applicable to data-aware nets), of which the first approach is called Simple Simulation. This boils down to constructing the automaton for each Declare constraint separately and updating them during execution. Some constraints, when intertwined, can lead to a permanent violation even when separately they do not. Hence, the full automaton of all individual constraints should be computed. This is called Smart Simulation. In this paper, we assume to execute mixed models of Declare and Petri nets as described in the latter approach. 
The choice for a dependency net and Declare constraints is founded on their

application in two very well-known and supported mining algorithms, namely Heuristics Miner [4] and Declare Miner [6]. The dependency net can be converted to Causal nets or Petri nets afterwards, as some examples in this paper show. Numerous techniques exist for this purpose and are incorporated in, e.g., Heuristics Miner. Declare models can be represented as a constraint set $\pi$ which is applied on activities $D$, thus resulting in $D M=\pi(D)$. A dependency net $D N$ is defined as a tuple of activities or nodes $L$ and the flow in the net $F=L \times L$, constituting a directed graph $D N=(L, F)$.

The different behavior of the models over activities $A$ can be represented as proposed in [I2]. Figure 미 shows the declarative behavior $\pi(D)$ over activities $D \subseteq A$ in the trapezoid-like, dotted shape and the procedural behavior $D N=$ $(L, F)$ in light gray with $L$ for the transitions, $L \subseteq A$. While the figure was proposed for modeling, it is now applied to mining. Therefore, the behavior contained in the log should be included. It is represented by the checkered, unstructured polygon.

We propose a mixed-paradigm model as follows: let MPM be a tuple $M P M=$ $(L, F, D, \pi)$. As such, we define both rules and strict sequences over the activities. The outcome of the model is then any part of $D N$ and $D M, M P M \subseteq$ $D N \cup D M$, which constricts the behavior of $\mathrm{A}$ in different ways:

- A more narrow result: $D N \cap D M$ : by taking the intersection of the behavior allowed by both models, it becomes possible to more strictly describe the process flow. This is represented by Figure W, where the intersection is indicated as the dark gray part. The main cause for this is that procedural models are good at capturing straightforward sequences, and Declare constraints have rich semantics to capture flexible behavior, but also long-distance relationships and duplicate tasks. Combining them offers the possibility to use the best representation in different situations.

- A mixed result: $D N \cup D M$ : by taking the union of all behavior, it becomes possible to capture behavior in the log that previously remained 


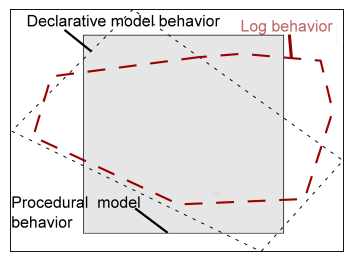

(a)

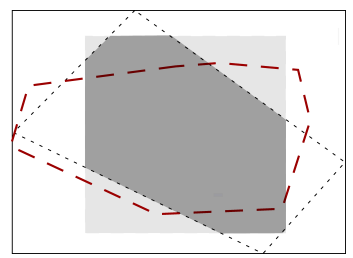

(b)

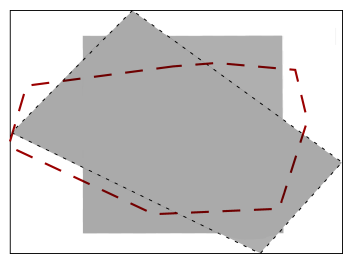

(c)

Figure 1: Graphical representation of the behavior allowed by the models, both procedural and declarative, and present in the log. In (b), both models restrict each others behavior to provide a stricter outcome. In (c), the union of both models is displayed. Any subsection can be an outcome of our mining approach.

undiscovered (typically in the procedural model) or was too broadly captured (typically in the declarative model). By taking subsets of this union, one can more closely retrieve the behavior in the log. This is shown in Figure ㅁ, where the possible subsets are indicated in dark gray.

The basic idea behind the mining algorithm explained below is the division of the set of activities $A$ in the event log into the two sets introduced above, namely the procedural activities $L$ and the so-called entropic activities $D$. We define entropic activities as activities which behavior is hard to capture in a strict procedural process flow. Arcs in mixed-paradigm models can be of the following type:

- $D D \subseteq D \times D$ : arcs between entropic activities are represented by Declare constraints.

- $D L \subseteq D \times L$ and $L D \subseteq L \times D$ : arcs between entropic and procedural 
activities are either explained by Declare constraints or the dependency net, with a prioritization of the former.

- $L L \subseteq L \times L$ : arcs between procedural activities are represented by the dependency net.

As such, there exist activities that are either completely captured in the Declare Model, $A_{D D} \in D$, only captured in the Petri net, $A_{L L} \in L$, and activities present in both models, $A_{D L_{D} / L D_{D}} \in D$ and $A_{D L_{L} / L D_{L}} \in L$. Note that $D=A_{D D} \cup A_{D L_{D}} \cup A_{L D_{D}}$ and $L=A_{L L} \cup A_{L D_{L}} \cup A_{D L_{L}}$. It is to be avoided that the paradigms become too convoluted, as the mining of both model types simultaneously is based on heuristics and cannot assure that there are no contradictions in the state spaces of both models. There exist model checking techniques, such as [25], which can convert both models into automata. The synchronous product could yield errors which can be taken into account during process discovery. However, the overhead introduced by these techniques is significant. Nonetheless, Fusion Miner actually exploits these "mismatches" in the state space, as both model types will restrict the workflow in a different way, yielding models with higher precision. This will become clear in the replay and

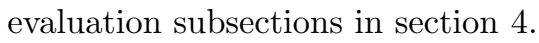

However, in order to keep both model types somewhat separated, Declare constraints are prioritized as mining and representation form, as the semantics of LTL formulae are richer than a dependency net relationship. Declare constraints are mined for all activities, except the ones in $A_{L L}$. If no constraints are discovered for an activity in $D$, the algorithm is resilient and puts the activity in $L$.

\subsection{The Fusion Miner algorithm}

The Fusion Miner implementation ${ }^{\mathbb{3}}$ is based on the combination of Heuristics Miner [4] and Declare Miner [26]. Starting from the dependency net, it identifies

\footnotetext{
${ }^{3}$ The implementation and high resolution figures can be found at j.processmining.be/fusionminer/.
} 


\begin{tabular}{|l||c|c|}
\hline Activities & $\mathbf{D}$ & $\mathbf{L}$ \\
\hline \hline $\mathbf{D}$ & $D \times D$ & $D \times L$ \\
& Declare constraints & $\begin{array}{c}\text { Declare Constraints } \\
(\text { Dependency graph })\end{array}$ \\
Contains & $A_{D D}$ & $A_{D L_{D}} \cup A_{D L_{L}}$ \\
\hline $\mathbf{L}$ & $L \times D$ & $L \times L$ \\
& $\begin{array}{c}\text { Declare Constraints } \\
(\text { Dependency graph })\end{array}$ & Dependency graph \\
Contains & $A_{L D_{D}} \cup A_{L D_{L}}$ & $A_{L L}$ \\
\hline
\end{tabular}

Table 1: The four different types of connections in a mixed-paradigm process model.

activities that are connected to a relatively higher number of other activities in the graph, as these can be considered a causal factor of the increase in potential process behavior. As such, we target them for inclusion in the set of activities that are subject to Declare mining in the second part of the discovery process. Finally, the Declare model can be pruned optionally and the mining result is displayed in a model containing both paradigms.

\subsubsection{The algorithm}

The algorithm starts off by calculating dependency measures for $\log L$ containing the activity alphabet $A_{L}$ with Heuristics Miner. This results in a tuple $(D G, D S, L 2 L, L D D)$ with $D G=A_{L} \times A_{L}$ the flow relations in the dependency net, $D S$ the direct succession values between $A_{L}, L 2 L$ the level 2-loop values, and $L D D$ long distance dependency values. By analyzing the strength of the direct succession metric (which is controlled by a threshold $d$ called Dependency threshold) between activities $A_{L}$, one can retrieve the activities most closely related in a small window containing local neighbors in the log. Activities that are connected to others many times, or are somewhat but not strongly connected, are candidates to be placed in the set of declarative activities $D \subseteq A_{L}$. Others that have few but strong connections to neighboring activities, are candidates to remain in the procedural part of the model $L \subseteq A_{L}$. Phrased differently, we target activities with unclear direct succession relations, which can be an indication of the ad-hoc all-over-the-place occurrence of this activity, which results 
in non-structured and cluttered up sequential process models. Note that this approach also often captures the activities that cannot be fitted into the model and thus puts tasks that are connected only when the "All activities connected" option is chosen in Heuristics Miner in $D$.

The details of the algorithm can be found below. It first checks for entropic activities and for this purpose we propose a metric called Activity Entropy $(A E)$ which captures the average of the direct succession $(D S)$ values between an activity and the others in the log where the dependency threshold $d$ is not met (lines 3-4). In other words, it captures weak dependencies. Procedural activities in a log will have a very low activity entropy, as most of the connections will be either strong $(>d)$ or non-existing (close or equal to zero). Based on a given threshold $0 \leq e \leq 1$ which is an input parameter of Fusion Miner, a proportion of the $\log$ is withheld. The different values $A E_{i}$ are ranked and $\left\lfloor\left|A_{L}\right|(1-e)\right\rfloor$ activities are kept in the sorted set $E$ (lines 5-6). Furthermore, if there is a gap of $1 / e$ between the values for $A E$ of two activities in $E$, the activities ranked below 255 the gap are removed (lines 7-9). This procedure avoids introducing too many activities in $D$ and as a consequence possibly too many constraints between them. Note, however, that a fully declarative model can be obtained by using 1 for $e$. Declare constraints mined for single activities are always included in the $\log$.

$260 e$ influences the discovery process thus in two ways. First of all, the higher $e$, the more activities will be put in $D$. Secondly, it will dampen the effect of considering activities as entropic when there is little evidence for this. Hence, the algorithm reacts differently to logs containing very procedural behavior, very flexible behavior, or a mixture of both. In the first case, $e$ will have less effect, where only higher values of $e(>0.5)$ will introduce many declarative activities (and constraints), which is reversely true for the second case. In the latter case, $e$ is the most sensitive, churning different results for the full interval of $[0,1]$. Therefore, one can use $e$ to determine the extent to which the log is flexible and change $e$ to retrieve different results. By first using a higher value of $e$ of over 0.7, the log will reveal the very entropic activities, and the user can change $e$ 


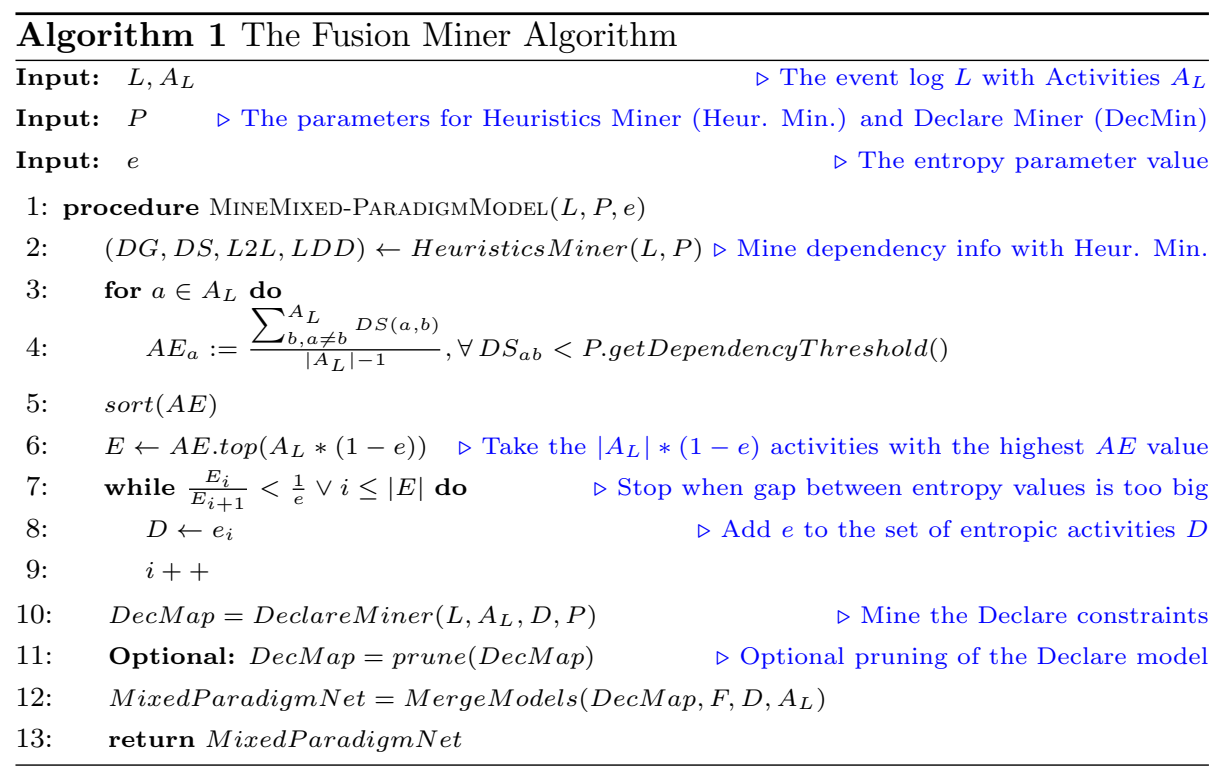

according to his needs.

\subsubsection{Pruning and Constraint Choice}

After the results of both miners are retrieved, an optional manipulation of the outcome is performed which cuts and adds some constraints, extending the approach proposed by [26]. (Alternate) Precedence and Response constraints (see Table 6) are transformed into Succession constraints when the antecedents and consequents involved appear exactly once, and Co-existence is removed when both activities appear at least once. The Alternate precedence constraints are reduced to Precedence when the consequent only appears once, etc. After this pruning phase, a check for transitivity is performed, as, for example, newly introduced Precedence constraints might be in a transitive relation with other Precedence constraints, while their superior Alternate Precedence predecessors were not.

Also, since the Declare constraint set contains over 20 entries and we com285 bine this set with a procedural model, some constraints become obsolete or less relevant:

- The Chain Response/Precedence/Succession() constraints are captured by 


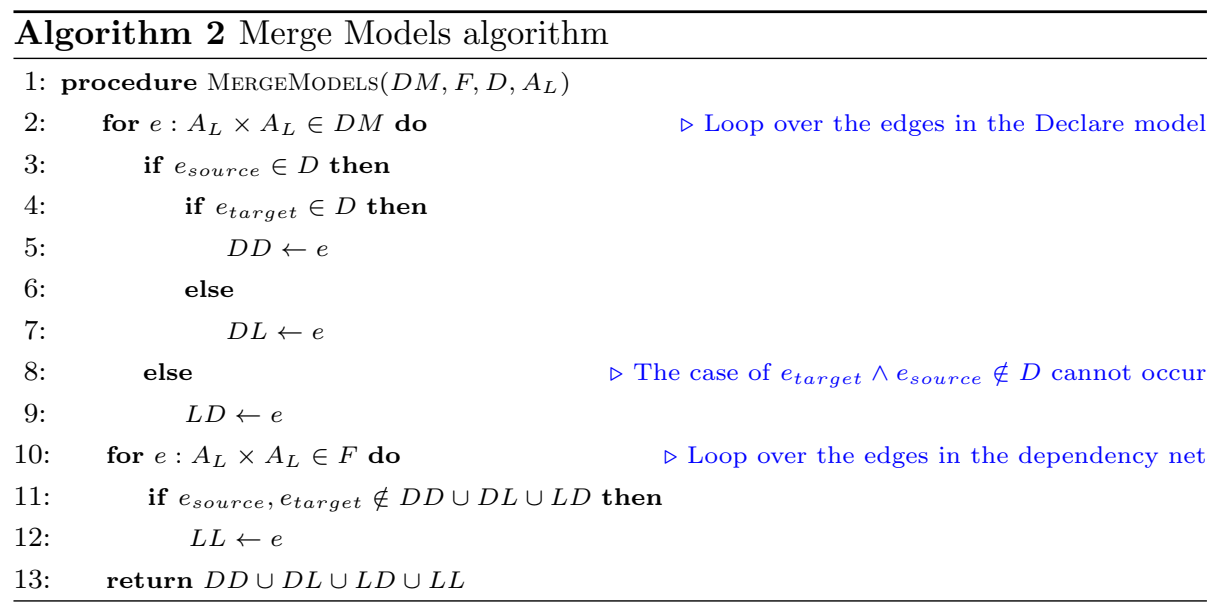

direct succession constraints in the procedural model.

- The Choice() constraints are captured by the XOR- and AND-joins and -splits in the procedural model. Furthermore, Declare constraints often implicitly invoke such constraints.

- Negative constraints are left out by default, but can be included. These constraint templates tend to introduce a lot of behavior as everything that is not supported is captured by these constraints, but often add little extra insight into the model.

\subsubsection{Performance and Noise}

The Fusion Miner algorithm utilizes two other algorithms that bound the performance. First of all, the very scalable Heuristics Miner algorithm is used, which is fast and resilient to noise [4]. Since every run of Fusion Miner mines the log fully with Heuristics Miner, it serves as a lower bound for calculation time (see $\mathbf{~}$, line 2), $t_{\text {HeurMin }}$. Through lines 3 to 9 , simple calculations are made which have a negligible effect on performance, with a complexity of $\frac{\left|A_{L}\right|\left(\left|A_{L}\right|-1\right)}{2}$, $t_{\text {DCalc }}$. Next, Declare Miner is applied. Depending on the size of $A E$, Declare Miner will calculate all binary constraints $\left(D=A_{L}\right)$ or only between $D$ and $D$ and $D$ and $P$. In the latter case, no constraints are mined for $P$ to $P$, which can reduce the calculation effort $t_{\text {DecMin }}$ significantly. Finally, the merging of both 
models is also linear to the number of arcs in the model (지), $t_{\text {MergMod }}$. Hence, the calculation time resides in between the interval $\left[t_{\text {HeurMin }}, t_{\text {HeuristicsMiner }}+\right.$ $\left.t_{D C a l c}+t_{\text {DecMin }}+t_{\text {MergMod }}\right]$.

Both algorithms have been proven to be capable of dealing with noise [ 8 , 27]. Most notably, Heuristics Miner has parameters that can be fine-tuned to disregard infrequent behavior and rare relationships by using the dependency threshold. Declare Miner can do the same by disregarding certain activities by using high confidence and percentage of instances settings [27]. Furthermore, the Fusion Miner algorithm also disregards infrequent behavior by pruning entropic activities (lines 6-9).

\subsection{Fitness and Precision of Mixed-Paradigm Models}

In order to fully assess the results of Fusion Miner, the fitness and precision of models derived from event logs should be evaluated to show how well the algorithm is capable of not only retrieving, but also narrowing down the behavior as described in the previous sections. Conformance metrics have been studied extensively, and numerous techniques exist. For a comprehensive overview of conformance metrics and techniques, the authors refer to [표].

As previously explained, Fusion Miner tries to fit the event log with different explanatory models. Hence, the overall fitness is often high, with a precision that outperforms techniques that are commonly used to achieve a fitness of 1 , such as ILP Miner [II]. Note that, when the Declare model is able to capture most of the flexible behavior which was hard to represent in a procedural net with a high Declare constraint support, the model will naturally end up with a high fitness value, oftentimes also reaching a perfect value of 1 .

The authors have implemented a preliminary fitness evaluation technique which tries to find an alignment of the log and the model. By traversing the state space, the model gets replayed in a best-first fashion. The technique creates the automaton of the Declare model (which is not as large as a Declareonly model when $e<1$ and due to pruning) and makes the product with the Petri net state space while traversing the trace during replay. Precision can 
be calculated during replay. The approach used here is based on artificially generated negative events, which was proposed by [1] and later improved by [29]. The authors propose a metric for precision which is called Behavioral Precision $p_{B}$. The basic setup consists of the generation of artificial negative events, which, simply put, are inserted when there can be no evidence found in the log for their occurrence at a certain trace index. The evidence is based on the prefix of the event, which can be configured to take into account a certain window size. Afterwards, the well-known precision evaluation of statistical and

\section{Experimental evaluation of Fusion Miner}

To illustrate the problems many traditional single-paradigm miners face, three examples are given below to illustrate where improvements can be achieved by Fusion Miner. Some basic metrics regarding the event logs used are provided in Table 2 . The first log contains a straightforward workflow which is distorted by the nearly random occurrence of an activity. The second example deals with a textbook case of a log with different layers of flexibility. The last one is a real-life $\log$, previously used in [ [ZZ, $\mathbb{Z}]$, which contains an incident-management process. The main insights are summarized at the end of this section in a list of contributions the Fusion Miner algorithm offers.

The first two logs are simulated using CPN Tools [24], which is able to execute mixed-paradigm models as indicated above. The logs were checked afterwards for temporary violations of Declare constraints and other issues that can arise, as explained in [피].

Mixed-paradigm models displayed in the text are represented as a combination of a dependency net and Declare constraints. However, the corresponding Petri net is also offered as an output. In the examples used in this paper, the following representation is used in the figures:

- The full arcs represent the procedural behavior as introduced by Heuristics Miner. They form the dependency net of the model. 


\begin{tabular}{|l|l|l|l|}
\hline Event Log & $\begin{array}{l}\text { Loosely con- } \\
\text { nected activ- } \\
\text { ity }\end{array}$ & PhD Process & $\begin{array}{l}\text { Incident Man- } \\
\text { agement Log }\end{array}$ \\
\hline Number of activities & 7 & 6 & 20 \\
Number of cases & 20 & 30 & 956 \\
Number of events & 786 & 402 & 9306 \\
Number of distinct traces & 10 & 30 & 212 \\
\hline
\end{tabular}

Table 2: Basic metrics describing the event log used for the evaluation of Fusion Miner.

- The checkered activities exceed the entropy threshold.

- The activities filled with a dark (red) shade fulfill the Exactly1 constraint.

- The activities filled in light gray fulfill the Existence constraints.

- The striped arrows represent ConDec constraints, which are labeled with the template's name.

- The squared activities represent activities fulfilling the Init and Last constraints.

\subsection{Example 1: A Loosely Connected Activity}

The first log of which the simulation model can be found in Figure 2 , represents a standard process with AND-split and -join which is repeated. Procedural discovery algorithms such as Alpha Miner and Heuristics Miner are perfectly capable of retrieving such procedural process behavior. However, introducing an activity which is only roughly tied to a strict position in the workflow such as $Z$, leaves the procedural miners guessing at its location in the process, as explained below. The activity is only connected to activity $C$ with a Precedence constraint, and to activity $D$ with an Alternate Precedence constraint.

\subsubsection{Issues with Currently Available Miners}

Heuristics Miner places $Z$ in a separate self-loop before the last activity, as can be seen in Figure 3 . The result of ILP Miner, depicted in Figure $\mathbb{\text { U }}$ recognizes 


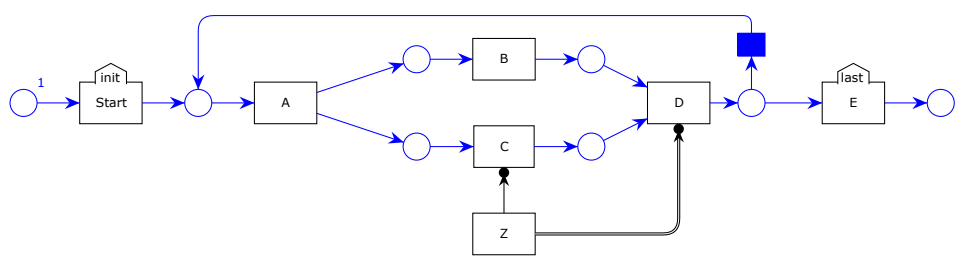

Figure 2: A Petri net containing a procedural workflow based around an AND-split and -join, extended with activity $Z$ which can occur in numerous positions in the process. It is connected to activity $C$ with a Precedence constraint, and to activity $D$ with an Alternate Precedence constraint.

曾.

\subsubsection{Discovery with Fusion Miner}

Fusion Miner is capable of mining the dependency net and merge it with Declare constraints. This way, it enlightens the unclear sequence information 


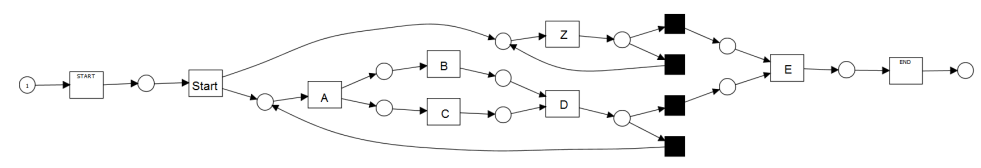

Figure 3: Result of Heuristics Miner (default settings) for the log of example 1.

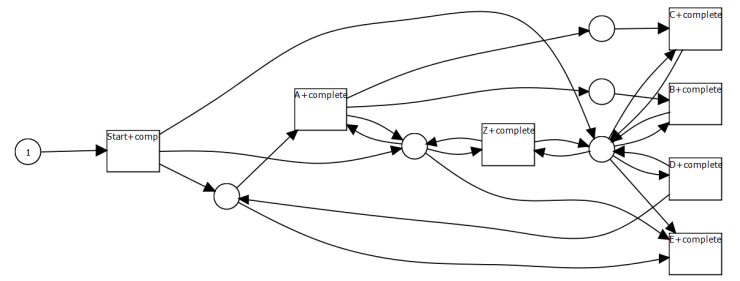

Figure 4: Result of ILP Miner (default settings) for the log of example 1.

very procedural behavior of the rest of the net, which was hidden in the Declare model due to the large amount of constraints. Much like the explaining of extra residual value in statistics, Fusion Miner tackles the unknown by fitting the data selectively by using characteristics of both process model types. In this example, the infinite loop enabling activity $Z$ all the time was better explained by introducing Declare constraints with Fusion Miner to reduce the state space of the model around $Z$, resulting in a more precise model as illustrated in Figure 6. The Petri net resulting from the dependency net in the mixed-paradigm model is shown in Figure $\square$.

\subsubsection{Fitness and Precision}

The results of Heuristics Miner, ILP Miner, Declare Miner, and Fusion Miner all fit the $\log 100 \%$. We selected a trace in the $\log$ that clearly shows where the result of Fusion Miner improves upon the three previous ones. The results for all miners are included and can be found in Table 3. The cases for which Fusion Miner will be more precise than the outcome of Heuristics Miner, are the appearance of $Z$ before $C$, due to the Precedence constraint and especially the appearance of $Z$ before $D$, due to the Alternate Precedence constraint. For 


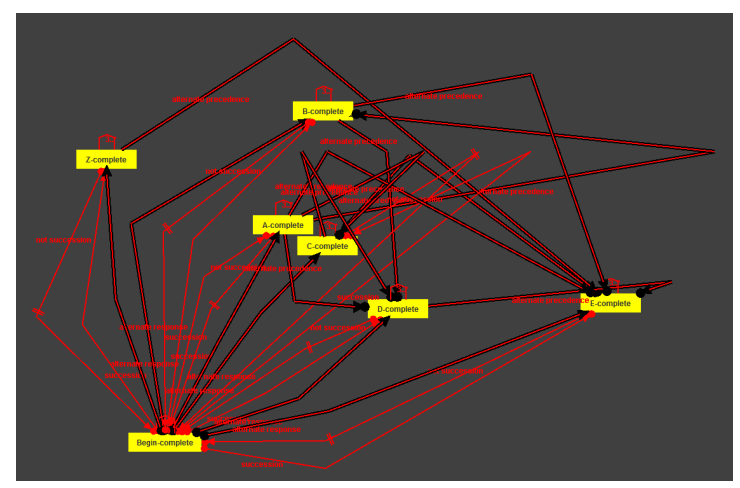

Figure 5: Result of Declare Miner for a support of $100 \%$ for the log of example 1. The Not Chain Succession constraints are excluded for readability.

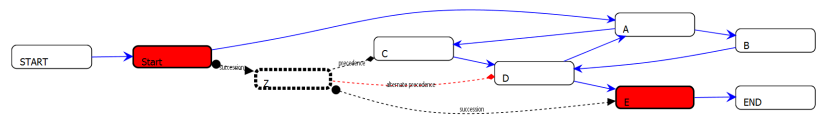

Figure 6: Result of Fusion Miner for example 1 with a support of $100 \%$ and $e=0.4$.

example, because $Z$ does not show up until just before the second $D$, there are less false positives compared to the Petri net. If we look at the behavioral precision scores, the following results are obtained: $p_{B_{H M}}=\frac{18}{32}=0.5625$, $p_{B_{I L P}}=\frac{19}{44}=0.4318$ and $p_{B_{F M}}=\frac{18}{30}=0.6$. A slight increase is achieved by using Fusion Miner, while improving vastly in terms of comprehensibility compared to Declare Miner. Since $Z$ occurs in an almost random fashion, there is not a huge gain in terms of precision, but Fusion Miner is still better capable of representing all the information that can be extracted from the log. Surprisingly, ILP Miner, known for its inclination towards fit but precise models, has

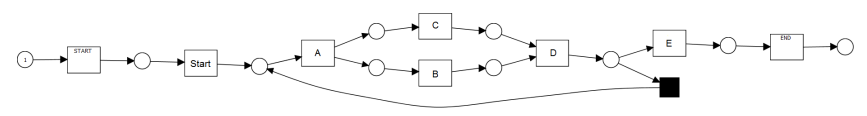

Figure 7: Petri net derived from the dependency net in Figure $\mathbf{G}$, containing the procedural behavior in the model. 
a lower precision value.

\begin{tabular}{|c|c|c|c|c|c|c|c|c|c|c|c|c|c|c|c|c|c|}
\hline \multirow{2}{*}{$\begin{array}{c}\text { Ind. } \\
1\end{array}$} & \multirow{2}{*}{$\begin{array}{l}\text { Act. } \\
\text { Start }\end{array}$} & \multicolumn{3}{|c|}{ Enabled MPM } & \multicolumn{3}{|c|}{ Enabled HM } & \multicolumn{4}{|c|}{ Enabled ILP } & \multicolumn{6}{|c|}{ Negative events } \\
\hline & & Start & & & Star & & & & & & & D & $\mathrm{E}$ & A & B & $\mathrm{C}$ & Z \\
\hline 2 & A & z & A & & A & $\mathrm{z}$ & & $\mathrm{z}$ & $\mathrm{A}$ & $\mathrm{D}$ & $\mathbf{E}$ & $\mathrm{D}$ & $\mathrm{E}$ & Start & B & $\mathrm{C}$ & \\
\hline 3 & z & z & B & & $\mathrm{z}$ & B & C & $\mathrm{z}$ & B & C & $\mathbf{D}$ & $\mathrm{D}$ & $\mathrm{E}$ & Start & A & $\mathrm{C}$ & \\
\hline 4 & C & Z & B & C & Z & B & C & Z & B & $\mathrm{C}$ & D & D & $\mathrm{E}$ & Start & A & B & \\
\hline 5 & $\mathrm{Z}$ & $\mathrm{z}$ & B & & $\mathrm{z}$ & B & & $\mathrm{z}$ & B & D & & D & $\mathrm{E}$ & Start & A & B & $\mathrm{C}$ \\
\hline 6 & B & $\mathbf{z}$ & B & & $\mathbf{z}$ & B & & Z & B & D & & D & $\mathrm{E}$ & Start & A & $\mathrm{C}$ & Z \\
\hline 7 & Z & Z & D & & Z & D & & Z & D & & & D & $\mathrm{E}$ & Start & A & B & C \\
\hline 8 & D & Z & D & & Z & D & & Z & $\mathrm{D}$ & & & $\mathrm{E}$ & Start & A & B & $\mathrm{C}$ & \\
\hline 9 & A & Z & A & E & Z & A & $\mathbf{E}$ & $\mathbf{z}$ & A & D & $\mathbf{E}$ & D & $\mathrm{E}$ & Start & B & $\mathrm{C}$ & Z \\
\hline 10 & B & $\mathbf{z}$ & B & C & $\mathbf{z}$ & B & $\mathrm{C}$ & $\mathbf{z}$ & B & C & $\mathbf{D}$ & D & $\mathrm{E}$ & Start & A & $\mathrm{C}$ & $\mathrm{Z}$ \\
\hline 11 & C & $\mathbf{z}$ & C & & $\mathbf{z}$ & $\mathrm{C}$ & & $\mathbf{z}$ & $\mathbf{D}$ & $\mathrm{C}$ & & D & $\mathrm{E}$ & Start & A & $\mathrm{B}$ & $\mathrm{Z}$ \\
\hline 12 & Z & $\mathrm{z}$ & & & $\mathrm{z}$ & $\mathbf{D}$ & & $\mathrm{Z}$ & $\mathbf{D}$ & & & $\mathrm{D}$ & $\mathrm{E}$ & Start & A & B & $\mathrm{C}$ \\
\hline 13 & D & z & D & & z & D & & z & A & $\mathrm{D}$ & $\mathbf{E}$ & $\mathrm{E}$ & Start & A & B & $\mathrm{C}$ & Z \\
\hline 14 & $\mathrm{E}$ & z & $\mathrm{E}$ & A & z & $\mathrm{E}$ & A & z & A & D & $\mathrm{E}$ & D & Start & A & B & $\mathrm{C}$ & Z \\
\hline
\end{tabular}

Table 3: The precision calculation for model 1 for all mining outcomes, based on artificially generated negative events. The false positives are indicated in bold. The mixed-paradigm model clearly enables less activities throughout the replaying of the trace, while still retaining maximal fitness.

\subsection{Example 2: PhD Process}

As another example, we provide the simple mixed-paradigm process model in Figure 8. Both Declare constraints and more sequentially-based Petri nets are combined to resemble the progress of a $\mathrm{PhD}$ student throughout his career, which contains the strict order of a first and second seminar followed by the defense. Meanwhile, he/she creates content which is subsequently published in journals or presented at a conference, resembled by the Alternate Precedence constraints. This constraint expresses that both Journal Paper and Conference can happen after Content Creation, and again only after the next occurrence of the Content Creation activity. The first seminar cannot happen before a first contribution to a conference and the second seminar has to be preceded by a journal publication. Note that, while the mixed-paradigm model is fairly 445 simple and understandable, the simulated event log presents characteristics that are typically found in real-life logs originating from complex processes. 


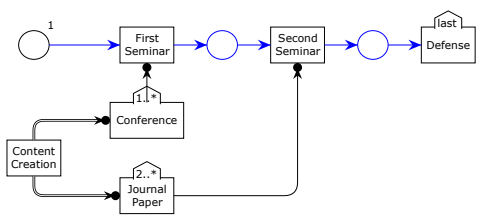

Figure 8: Workflow with different layers of flexibility representing the progress of a $\mathrm{PhD}$ student.

\subsubsection{Issues with Currently Available Miners}

If one mines the $\log$ with Heuristics Miner, the algorithm is unable to retrieve the exact position and relation of the three activities Content Creation, Conference and Journal Paper as shown in Figure 9 . While Heuristics Miner captures loops and invisible events to support the quite random appearance of Content Creation, it fails to capture the relation of Journal Paper and Second Seminar. Furthermore, the model is cumbersome to read due to the large number of invisible tasks needed to express the flexible nature of the relations.

Note that for Heuristics Miner it is possible to use other configurations, e.g. one with a lower dependency threshold. This would result in a model that better captures the behavior in the event log, but this solution would include a very generic model in which every transition can be executed in any order. Flexible parts of a log that are not captured (well) by procedural models (as they remained either too restrictive or too general) can be represented with declarative constraints to retrieve them in a more correct and readable way. Although capturing flexible behavior might be possible with procedural models, the sequential information would end up in a very convoluted and unstructured graph of loops, splits and joins, and arrows pointing every direction due to the ad-hoc appearance of activities as can be seen in Figure $\mathbf{\text { Q }}$. Since most Declare constraints represent behavior that can be labeled as non-trivial token games, they are better able to represent such parts of an event log. For example, expressing Alternate Precedence in a Petri net is a challenging task, leading to the usage of artificial model constructs to approximate the same state space.

Again, Declare Miner mines a fitting (for a support of 100\%) model with 
low comprehensibility. The result is shown in Figure [0]. The technique of [23] puts Defense on a lower hierarchical level, and thus is not able to recognize the flexible activities.

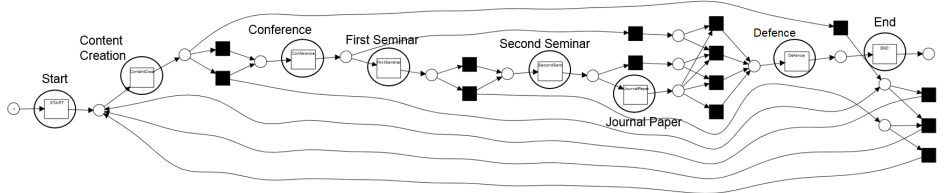

Figure 9: Result of Heuristics Miner for $\log 2$ in Figure $\mathbf{8}$ (Default settings and with reduced invisible activities).

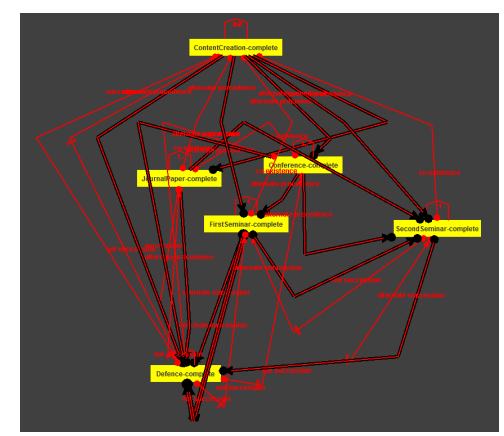

Figure 10: Result of Declare Miner for $\log 2$. The support used is $100 \%$.

\subsubsection{Discovery of the PhD process with Fusion Miner}

Figures $\square$ and $\square 2$ show the discovered mixed-paradigm models in ProM. An overview of the different activity entropy values can be found in Table $⿴$. Even for a small entropy value $e=0.2$, and due to the $A_{L} *(1-e)$ pruning step, and a constraints support of $100 \%$, the activity Content Creation becomes subject to Declare constraint mining (Figure $\square$ ). By its constant enabledness it can appear anywhere in the workflow and clutter up a sequential process. By retrieving a few constraints for the activity, we are able to represent it in a sense-making way in a mixed model. The model is already capable of capturing the initial model more correctly, as the relationships between ContentCreation and the other activities are correct. The arc between SecondSeminar and JournalPaper 


\begin{tabular}{|l||l|l|l|}
\hline Activity & Activity Entropy & Eligible for $e=0.2$ & Eligible for $e=0.5$ \\
\hline Content Creation (CC) & 0.561 & Yes & Yes \\
Journal Paper (JP) & 0.557 & No & Yes \\
Conference (CO) & 0.52 & No & Yes \\
Second Seminar (SS) & 0.38 & No & No \\
First Seminar (FS) & 0.242 & No & No \\
Defense (DE) & 0 & No & No \\
\hline
\end{tabular}

Table 4: The activity entropy values for the $\mathrm{PhD}$ example to illustrate the workings of the algorithm. For $e=0.2$, only $C C$ is taken into account, due to the $A_{L} *(1-e)$ pruning step of $A E$. For $e=0.5, J P$ and $C O$ are also included in $D$.

485 is still incorrect.

By raising the entropy level (Figure ए2), more activities are added to the declarative set $D$, in this case Conference and Journal Paper. This makes sense given the model. Only constrained by the appearance of Content Creation, these activities are also rather unpredictable. Note that the procedural part of the model is becoming smaller and smaller, while the Declare constraints offer the same behavior and more.

If we position this approach in Figure $\mathbf{~}$, it would be categorized as an attempt to mine behavior more closely by retrieving the intersection of both outcomes, depicted in Figure एb.

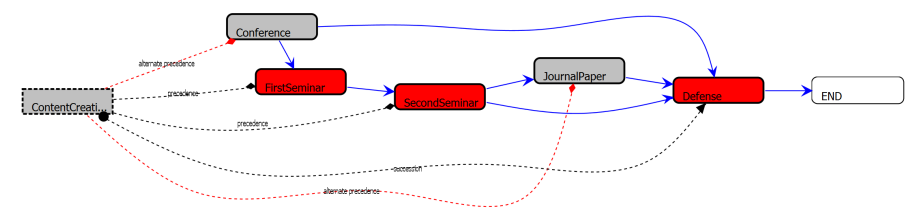

Figure 11: Result of Fusion Miner with $e=0.2$ for $\log 2$. The activity on the left is the only entropic one and is connected with Declare constraints to the other five. The arrow between Second Seminar and Journal Paper is still incorrect, but the Alternate precedence constraints are better capable of capturing the behavior in a clear way.

In Table 5, the second result (for $e=0.5$ ) of Fusion Miner (represented as Declare and Petri net in Figure ए.3) is compared to ILP Miner's result for the 


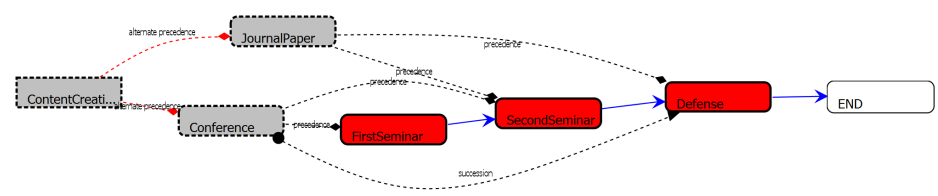

Figure 12: Result of Fusion Miner with $e=0.5$ for $\log 2$. When raising the entropy level in the miner, Conference and Journal Paper get included in the declarative part of the model. The relations between the activities are now all captured correctly.

$\mathrm{PhD}$ example, which can be found in Figure [4]. ILP Miner is chosen as it generally guarantees a fitness of 1 . Again, the false positives are indicated in bold. Notice that, even for a dependency measure of 0, Heuristics Miner was unable to derive a perfectly fitting model for the event log and is not used in the comparison.

For this trace in particular, the precision scores are $p_{B_{I L P}}=\frac{22}{13+22}=0.629$ and $p_{B_{M P M}}=\frac{19}{19+5}=0.792$ for $e=0.5$.

As shown in Table 15, it is clear that ILP Miner's Petri net keeps a lot more activities enabled throughout the replay of the trace, while Fusion Miner's result enables less activities and thus triggers less false positives, resulting in a higher precision value. Note that, since this trace is short, the prefix window for negative event generation is small, resulting in artificial negative event generation only after some activities, punishing ILP Miner only towards the end. For longer traces, ILP Miner's outcome would produce even more false positives throughout the trace. Compared to the first example, it is clear that Fusion Miner outperforms traditional procedural mining algorithms especially for logs with different layers of flexibility.

The result of Declare Miner, as shown in Figure [0, also has a fitness of 1 and a high precision, but the comprehensibility is really low. Furthermore, while Declare models usually aim for high flexibility, the outcome of this model is very strict, surpassing the purpose of Declare. Finally, the precision result of Fusion Miner as depicted in Table 1 is the same as the one of Declare Miner, as the resulting Declare model enables the same activities as the mixed-paradigm 
model during replay.

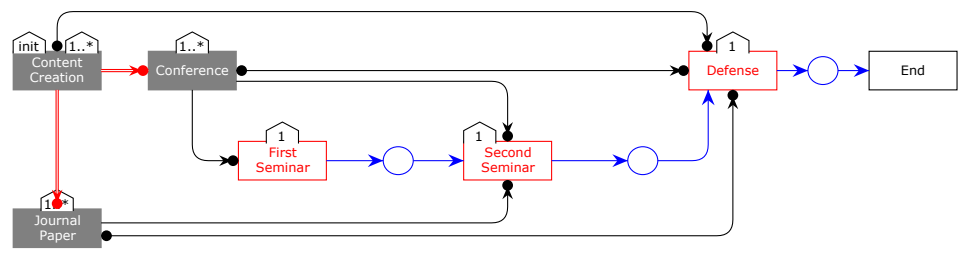

Figure 13: Mixed-paradigm model with $e=0.5$ from Figure 미, for which the dependency net is translated into a Petri net.

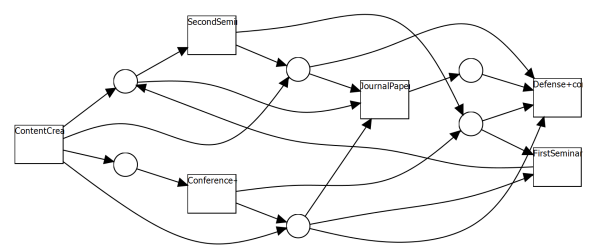

Figure 14: Result of ILP Miner for the PhD example log (default settings).

\subsection{The Incident Management Log}

This real-life example contains different steps of an incident management process, which starts off with the assignment of a help desk resource with Status Assigned. This activity can be succeeded by numerous other activities, such as the installment of a Status Work In Progress status, a Pending status, or the use of a special resource such as Group GPC CORK or Group ORS MS BACKOFFICE.

\subsubsection{Issues with Currently Available Miners}

The result of Heuristics Miner with default settings is given in Figure $\mathbf{T 5}^{\mathbb{P}}$. The dependency net is large, as it tries to fit most of the log variants, which results in a spaghetti-like model. The result of Declare Miner is not included, as it was unreadable due to the enormous amount of constraints (over 100

\footnotetext{
${ }^{4} \mathrm{~A}$ high-resolution version of the figure can be found at $\mathrm{j}$.processmining.be/fusionminer
} 


\begin{tabular}{|c|c|c|c|c|c|c|c|c|c|c|c|c|c|c|c|c|}
\hline \multirow{2}{*}{$\begin{array}{c}\text { Ind. } \\
1\end{array}$} & \multirow{2}{*}{$\begin{array}{c}\text { Act. } \\
\mathrm{CC}\end{array}$} & \multicolumn{6}{|c|}{ Enabled ILP } & \multicolumn{4}{|c|}{ Enabled MPM } & \multicolumn{5}{|c|}{ Negative events } \\
\hline & & $C C$ & & & & & & $C C$ & & & & $\mathrm{CO}$ & JP & FS & SS & $\mathrm{DE}$ \\
\hline 2 & $\mathrm{CO}$ & $\mathrm{CC}$ & $\mathrm{CO}$ & $\mathrm{JP}$ & SS & & & $\mathrm{CC}$ & $\mathrm{CO}$ & JP & & & & & & \\
\hline 3 & JP & $\mathrm{CC}$ & $J P$ & FS & SS & & & $\mathrm{CC}$ & $J P$ & FS & & $\mathrm{CO}$ & & & & \\
\hline 4 & $\mathrm{CC}$ & $C C$ & FS & & & & & $C C$ & FS & & & $\mathrm{CO}$ & JP & & & \\
\hline 5 & $\mathrm{CO}$ & $\mathrm{CC}$ & $\mathrm{CO}$ & JP & FS & ss & DE & $\mathrm{CC}$ & $\mathrm{CO}$ & JP & FS & SS & & & & \\
\hline 6 & FS & $\mathrm{CC}$ & JP & $F S$ & ss & DE & & $\mathrm{CC}$ & $\mathrm{JP}$ & $F S$ & & $\mathrm{CO}$ & SS & $\mathrm{DE}$ & & \\
\hline 7 & SS & $\mathrm{CC}$ & $\mathbf{J P}$ & FS & $S S$ & DE & & $\mathrm{CC}$ & JP & $S S$ & & $\mathrm{CC}$ & $\mathrm{CO}$ & JP & FS & $\mathrm{DE}$ \\
\hline 8 & JP & $\mathrm{CC}$ & $J P$ & FS & ss & DE & & $\mathrm{CC}$ & $J P$ & DE & & $\mathrm{CC}$ & $\mathrm{CO}$ & FS & SS & $\mathrm{DE}$ \\
\hline 9 & $\mathrm{DE}$ & $\mathrm{CC}$ & FS & $D E$ & & & & $\mathrm{CC}$ & $D E$ & & & $\mathrm{CC}$ & $\mathrm{CO}$ & $\mathrm{JP}$ & FS & ss \\
\hline
\end{tabular}

Table 5: The precision calculation for the PhD example for ILP Miner (default settings) and Fusion Miner. As can be seen in the Enabled columns, there are a lot of activities enabled all the time in the Petri net of ILP Miner, resulting in lower precision, which is punished by the high false positive rate (negative events indicated in bold). Fusion Miner's model enables much less activities while retaining the same fitness value. The abbreviations of the activities can be found in Table .

constraints for a support of $100 \%$ and 150 for a support of $75 \%$ ), which cannot be represented easily in a process map. Furthermore, it did not contain a lot of sequential information, such as (Alternate) Precedence/Succession results. Declare Miner oftentimes has a hard time dealing with real-life logs, which is supported by the fact that UnconstrainedMiner (correctly) found numerous such constraints. However, the result of UnconstrainedMiner includes all supported constraints, hence not a Declare model for a certain support.

\subsubsection{Discovery with Fusion Miner}

Fusion Miner depends on Declare Miner's result, which did not include many Declare constraints kept for mining in the Fusion Miner algorithm. However, by using various Declare constraint support values, it becomes possible to quickly derive various well- and less-supported flows in the dependency net. The result shown in Figure $]^{\boxplus}$ for a support of $75 \%$ and $e=0.5$, shows many activities only executed once. In combination with the Succession constraints which yield the obligatory execution of the consequent, the main flow can be read from the figure. This highlights another benefit of using Fusion Miner: scrutinizing the $\log$ for frequent and less frequent workflow behavior. 


\subsubsection{Fitness and Precision}

Since the model does not fit the log for $100 \%$, the replaying algorithm cannot enact the model as the move-on-model and move-on-log concept are not integrated yet. Through visual inspection, however, it was clear that for certain Declare constraint support values, the result was very precise, as the combination of Exactly1 and Succession constraints clearly delineated the workflow.

In general, Fusion Miner can cope with real-life and complex logs by building on top of two robust algorithms that can deal with noise (as explained in Section 3.2.3) and are always capable of retrieving a somewhat fitting model (Heuristics Miner) and a model with a varying level of fitness (Declare Miner through its support parameter). Furthermore, real-life logs often consist of behavior that is at least somewhat flexible but still contains some structured sequences. In other cases, Fusion Miner can used with settings that lean towards procedural and declarative by using parameter $e$. Furthermore, Fusion Miner will never come up with more convoluted models, which follows from the way the models are merged. The number of arcs found by Heuristics Miner and the fact that Declare constraints are hierarchical, provide an upper bound for the number of arcs found by Fusion Miner.

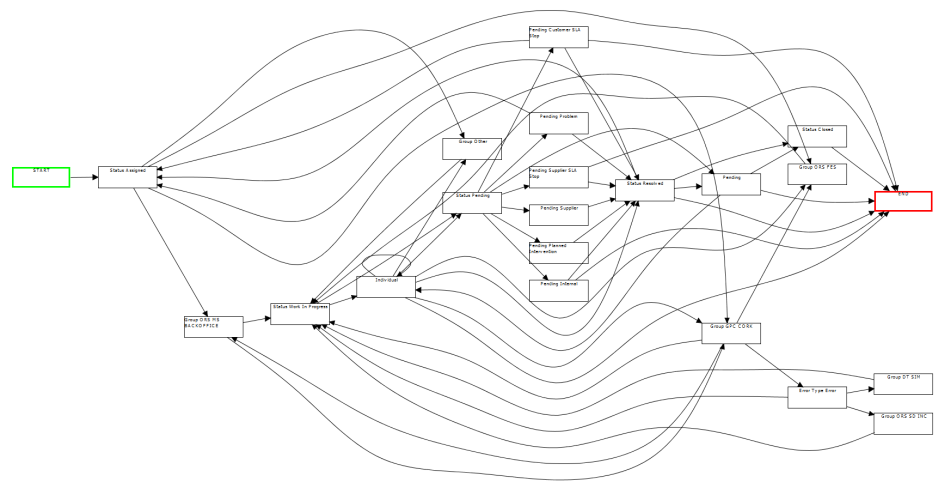

Figure 15: The result of Heuristics Miner (default settings) for the real-life log. The model displays most of the process variants, which results in a convoluted model in which a main process flow is hard to discern. 


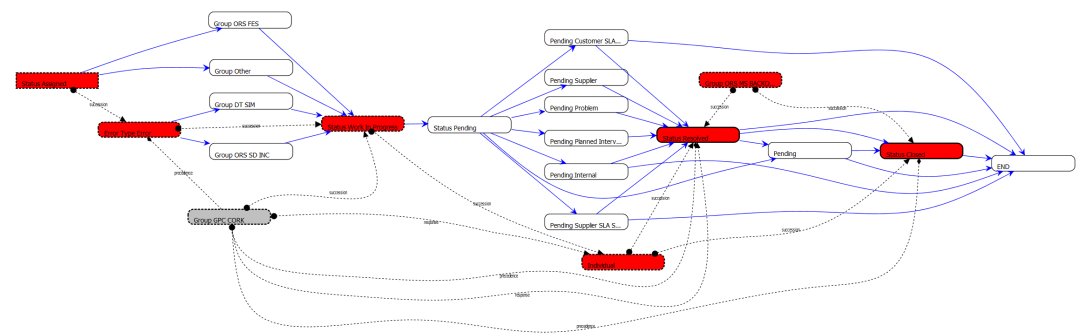

Figure 16: The mixed-paradigm result of Fusion Miner for the real-life example with $e=0.5$ and a support of $75 \%$. The main flow is clearly visible through the main Declare constraints, model variants can be deduced from the remaining procedural process constructs.

\subsection{Summary}

The previous examples have shown the main issues that traditional miners face when dealing with different layers of flexibility. The benefits that can be provided by Fusion Miner and its main use cases are:

- More precise models: Figure $\mathbb{1 6}$ shows how a Declare model can cut off parts of a procedural model, resulting in a more precise model. Fusion Miner exploits this intersection because:

- Declare constraints have different semantics that are better suited to capture flexible behavior. They provide, e.g., richer semantics for loops (e.g. the Alternate Precedence constraint) and duplicate tasks.

- Declare constraints are derived via constraint support on trace level. As such, they are, reinforced by their non-local sequence semantics, better capable of capturing long-distance relationships. Furthermore, the are mined for a certain support level which ensures the presence of the constraint, while many procedural miners use heuristics which cannot guarantee the end result to be a true reflection of the behavior in the event log.

There often exists a trade-off between precision and generalization [28]. In the case of Fusion Miner, the option for more precise rather than general 
models was made. However, more general models can be mined by lowering dependency thresholds in Heuristics Miner, and adding less constraints such as Alternates to the constraint set in Declare Miner.

- More fitting models: As illustrated in paragraph [.], Declare models often capture only the rough part of the work flow, depending on the support level they are granted and the templates that are used. While procedural models such as Petri nets can be relaxed to include more behavior by introducing extra connections and, e.g., invisible activities, resulting in an overly general model, Declare constraints can still capture rough or even very fitting information about the parts of the log that are rather flexible and would result in a very generic procedural model. Figure 미 illustrates this as the dark gray mixed model which represents the combination of a procedural and declarative model can cover extra parts where the light gray procedural model was not able to go.

By combining both, thus using Declare constraints where flexibility is needed while retaining procedural fragments, Fusion Miner can keep a high fitness value by relaxing procedural models with (precisely) fitting Declare constraints.

- More comprehensible models: Model comprehension is influenced by numerous factors [31, 32]. By combining paradigms suited for different granularities of flexibility, mixed-paradigm models are capable of representing processes in the form most suited to their nature. For example, parts of an event log which would typically be captured by a Petri net containing invisible activities, can perhaps better be explained by using some Declare constraints. Furthermore, Declare models can be presented in a simpler way for readers with procedural constructs which offer stricter models where possible. As shown in the previous examples, mined Declare models are often incomprehensible and use a vast amount of constraints of which many are redundant. 
The basic idea boils down to the exploitation of the stronger semantics of Declare constraints in procedural models in order to introduce more flexibility, while maintaining a procedural part in which strict sequences are still present where the process in the log does not need extra flexibility. Very loose or very procedural logs are not the target of the approach, but can be mined by choosing the correct settings. E.g., by choosing an entropy level $e$ of 0 or 1 , one mines a fully procedural or fully declarative process model respectively. As such, Fusion Miner provides a framework to incorporate any level of flexibility, which can also be used for log exploration.

The three pillars of process mining are supported as follows. First of all, Fusion Miner can contribute by discovering a new type of model that combines constraints and sequences. The results can be crosschecked with manager's believe of current as-is models. This gives rise to a new set of conformance checking techniques, which might include pinpointing non-fitting or imprecise behavior to either the procedural part or declarative part. Based on this, changes can be made to current models in place in the information system focusing on, e.g., certain constraints or certain sequences.

\section{Conclusions and Future Work}

In this paper, the authors address the challenge of mixed-paradigm process mining. Fusion Miner was proposed, an algorithm to mine for procedural and declarative process model constructs simultaneously to represent workflow behavior in an event log. Results show that this approach yields more precise models, especially in environments with multiple layers of flexibility, and more comprehensive models, as recapitulated in Section t.4.

Future work includes a more in-depth approach to compliance, as a preliminary replaying algorithm is used for conformance checking. Furthermore, the possibility of applying other declarative process model algorithms should be investigated, as recent technique have vastly improved in terms of perfor- 
constraint support) used in the models can be further investigated. Also, the approach used for deciding upon the inclusion of activities in set $D$ could be elaborated more extensively, as numerous other valid ways such as activity clustering, pattern recognition, etc., might yield different and possibly better results. capture constraints and fixed procedures throughout multiple layers of a process $\log$, similar to [2:3].

Acknowledgments.

This research is funded by FWO (Fonds voor Wetenschappelijk Onderzoek) Project G0804 13N.

\section{References}

[1] W. M. van der Aalst, Process Mining: Discovery, Conformance and Enhancement of Business Processes, Springer, 2011.

[2] R. Gopal, J. R. Marsden, J. Vanthienen, Information mining - reflections on recent advancements and the road ahead in data, text, and media mining, Decision Support Systems 51 (4) (2011) 727-731.

[3] W. M. van der Aalst, T. Weijters, L. Maruster, Workflow mining: Discovering process models from event logs, Knowledge and Data Engineering, IEEE Transactions on 16 (9) (2004) 1128-1142.

[4] A. Weijters, W. M. van der Aalst, A. A. De Medeiros, Process mining with the heuristics miner-algorithm, TU Eindhoven, Tech. Rep. 166.

[5] T. Murata, Petri nets: Properties, analysis and applications, Proceedings of the IEEE 77 (4) (1989) 541-580.

[6] F. M. Maggi, R. J. C. Bose, W. M. van der Aalst, Efficient discovery of understandable declarative process models from event logs, in: Advanced Information Systems Engineering, Springer, 2012, pp. 270-285. 
[7] J. De Smedt, J. De Weerdt, J. Vanthienen, Multi-paradigm process mining: retrieving better models by combining rules and sequences, in: On the Move to Meaningful Internet Systems, Springer, 2014, pp. 446-453.

675 [8] J. De Weerdt, M. De Backer, J. Vanthienen, B. Baesens, A multidimensional quality assessment of state-of-the-art process discovery algorithms using real-life event logs, Information Systems 37 (7) (2012) 654-676.

[9] S.-Y. Hwang, W.-S. Yang, On the discovery of process models from their instances, Decision Support Systems 34 (1) (2002) 41-57.

[10] C. W. Günther, W. M. van der Aalst, Fuzzy mining-adaptive process simplification based on multi-perspective metrics, in: Business Process Management, Springer, 2007, pp. 328-343.

[11] J. M. E. van der Werf, B. F. van Dongen, C. A. Hurkens, A. Serebrenik, Process discovery using integer linear programming, in: Applications and Theory of Petri Nets, Springer, 2008, pp. 368-387.

[12] M. Pesic, H. Schonenberg, W. M. van der Aalst, Declare: Full support for loosely-structured processes, in: Enterprise Distributed Object Computing Conference, IEEE, 2007, pp. 287-287.

[13] C. Di Ciccio, M. Mecella, A two-step fast algorithm for the automated discovery of declarative workflows, in: Computational Intelligence and Data Mining (CIDM), 2013 IEEE Symposium on, IEEE, 2013, pp. 135-142.

[14] M. Westergaard, C. Stahl, H. A. Reijers, Unconstrainedminer: Efficient discovery of generalized declarative process models.

[15] F. Chesani, E. Lamma, P. Mello, M. Montali, F. Riguzzi, S. Storari, Exploiting inductive logic programming techniques for declarative process mining, in: Transactions on Petri Nets, Springer, 2009, pp. 278-295.

[16] Y.-H. Hu, Y.-L. Chen, Mining association rules with multiple minimum supports: a new mining algorithm and a support tuning mechanism, Decision Support Systems 42 (1) (2006) 1-24. 
[23] F. M. Maggi, H. A. Reijers, T. Slaats, The automated discovery of hybrid processes, in: Business Process Management, 2014.

[24] M. Westergaard, CPN Tools 4: multi-formalism and extensibility, in: Application and Theory of Petri Nets and Concurrency, Springer, 2013, pp. 400-409.

[25] A. Duret-Lutz, D. Poitrenaud, Spot: an extensible model checking library using transition-based generalized büchi automata, in: MASCOTS, IEEE, 2004, pp. $76-83$.

[26] F. M. Maggi, R. J. C. Bose, W. M. van der Aalst, A knowledge-based inteInformation Systems Engineering, Springer, 2013, pp. 433-448. 
[27] F. M. Maggi, A. J. Mooij, W. M. van der Aalst, User-guided discovery of declarative process models, in: Computational Intelligence and Data Mining (CIDM), 2011 IEEE Symposium on, IEEE, 2011, pp. 192-199.

[28] S. vanden Broucke, J. De Weerdt, J. Vanthienen, B. Baesens, A comprehensive benchmarking framework for conformance analysis between procedural process models and event logs in prom, in: CIDM, IEEE, 2013, pp. 254-261.

[29] S. vanden Broucke, J. De Weerdt, J. Vanthienen, B. Baesens, Determining process model precision and generalization with weighted artificial negative events, IEEE TKDE 26 (8) (2013) 1877-1889.

[30] C. Haisjackl, I. Barba, S. Zugal, P. Soffer, I. Hadar, M. Reichert, J. Pinggera, B. Weber, Understanding declare models: strategies, pitfalls, empirical results, Software \& Systems Modeling 1-28.

[31] J. Mendling, M. Strembeck, J. Recker, Factors of process model comprehensionfindings from a series of experiments, Decision Support Systems 53 (1) (2012) 195-206.

[32] K. Figl, J. Recker, J. Mendling, A study on the effects of routing symbol design on process model comprehension, Decision Support Systems 54 (2) (2013) 1104-1118. 
Table 6: An overview of Declare constraint templates.

\begin{tabular}{|c|c|c|}
\hline Template & LTL Formula & Description \\
\hline Existence $(A)$ & $\diamond A$ & Activity A appears at least once. \\
\hline Existence $(A, n)$ & $\diamond(A \wedge \bigcirc($ existence $(n-1, A)))$ & Activity A appears at least $n$ times. \\
\hline Absence $(A, n)$ & $\neg$ existence $(n, A)$ & $\begin{array}{l}\text { Activity A can happen at most } n \\
\text { times. }\end{array}$ \\
\hline $\operatorname{Exactly}(A, n)$ & existence $(n, A) \wedge$ absence $(n+1, A)$ & $\begin{array}{l}\text { Activity A has to be executed at least } \\
n \text { times. }\end{array}$ \\
\hline $\operatorname{Init}(A)$ & $A$ & $\begin{array}{l}\text { Each instance has to start with activ- } \\
\text { ity A. }\end{array}$ \\
\hline Last $(A)$ & $\square(A \Rightarrow \neg X \neg A)$ & $\begin{array}{l}\text { Each instance has to end with activity } \\
\text { A. }\end{array}$ \\
\hline $\begin{array}{l}\text { Responded } \\
\text { existence }(A, B)\end{array}$ & $\diamond A \Rightarrow \diamond B$ & $\begin{array}{l}\text { If } \mathrm{A} \text { happens at least once then } \mathrm{B} \text { has } \\
\text { to happen or happened before } \mathrm{A} \text {. }\end{array}$ \\
\hline Co- existence $(A, B)$ & $\diamond A \Leftrightarrow \diamond B$ & $\begin{array}{l}\text { If } \mathrm{A} \text { happens then } \mathrm{B} \text { has to happen or } \\
\text { happened after } \mathrm{A} \text { and vice versa. }\end{array}$ \\
\hline Response $(A, B)$ & $\square(A \Rightarrow \diamond B)$ & $\begin{array}{l}\text { Whenever activity } \mathrm{A} \text { is executed, ac- } \\
\text { tivity } \mathrm{B} \text { has to be executed afterwards } \\
\text { eventually. }\end{array}$ \\
\hline Precedence $(A, B)$ & $(\neg B U A) \vee \square(\neg B)$ & $\begin{array}{l}\text { Activity } \mathrm{B} \text { has to be preceded by ac- } \\
\text { tivity A. }\end{array}$ \\
\hline Succession $(A, B)$ & response $(A, B) \wedge$ precedence $(A, B)$ & $\begin{array}{l}\text { Both Response(A,B) and Prece- } \\
\text { dence(A,B) hold. }\end{array}$ \\
\hline $\begin{array}{l}\text { Alternate } \\
\text { precedence }(A, B)\end{array}$ & $\begin{array}{l}\text { precedence }(A, B) \quad \wedge \square(B \quad \Rightarrow \\
\bigcirc(\text { precedence }(A, B))\end{array}$ & $\begin{array}{l}\text { Activity B cannot happen before } \mathrm{A} \\
\text { After it happens, it cannot happen be- } \\
\text { fore the next } \mathrm{A} \text { again. }\end{array}$ \\
\hline $\begin{array}{l}\text { Alternate } \\
\text { response }(A, B)\end{array}$ & $\square(A \Rightarrow \bigcirc(\neg A U B))$ & $\begin{array}{l}\text { After each activity } A \text {, at least one ac- } \\
\text { tivity } B \text { is executed. Another A can } \\
\text { be executed again only after the first } \\
\text { B. }\end{array}$ \\
\hline $\begin{array}{l}\text { Alternate } \\
\text { succession }(A, B)\end{array}$ & $\begin{array}{lr}\text { alternate } & \text { response }(A, B) \\
\wedge \text { alternate } & \text { precedence }(A, B)\end{array}$ & $\begin{array}{l}\text { Both alternate precedence }(\mathrm{A}, \mathrm{B}) \text { and } \\
\text { alternate response }(\mathrm{A}, \mathrm{B}) \text { hold. }\end{array}$ \\
\hline $\begin{array}{l}\text { Chain } \\
\text { precedence }(A, B)\end{array}$ & $\square(\bigcirc B \Rightarrow A)$ & $\begin{array}{l}\text { B can get executed only directly after } \\
\text { A. }\end{array}$ \\
\hline Chain response $(A, B)$ & $\square(A \Rightarrow \bigcirc B)$ & After A the next activity has to be B. \\
\hline $\begin{array}{l}\text { Chain } \\
\text { succession }(A, B)\end{array}$ & $\square(A \Leftrightarrow \bigcirc B)$ & $\begin{array}{l}\text { A and B can happen only next to each } \\
\text { other. }\end{array}$ \\
\hline $\begin{array}{ll}\text { Not } & c o- \\
\text { existence }(A, B) & \\
\end{array}$ & $\neg(\diamond A \wedge \diamond B)$ & $\begin{array}{l}\text { Only one of the two tasks A or B can } \\
\text { be executed, but not both. }\end{array}$ \\
\hline Not succession $(A, B)$ & $\square(A \Rightarrow \neg(\diamond B))$ & $\begin{array}{l}\text { Before B there cannot be } \mathrm{A} \text { and after } \\
\text { A there cannot be B. }\end{array}$ \\
\hline $\begin{array}{l}\text { Notchain } \\
\text { succession }(A, B)\end{array}$ & $\square(A \Rightarrow \bigcirc(\neg B))$ & $\begin{array}{l}\mathrm{A} \text { and } \mathrm{B} \text { can never get executed next } \\
\text { to each other where } \mathrm{A} \text { is executed first } \\
\text { and B second. }\end{array}$ \\
\hline Choice $(A, B)$ & $\diamond A \vee \diamond B$ & At least one A or B has to be executed. \\
\hline $\begin{array}{l}\text { Exclusive } \\
\text { choice }(A, B)\end{array}$ & $(\diamond A \vee \diamond B) \wedge \neg(\diamond A \wedge \diamond B)$ & A or B has to happen, but not both. \\
\hline
\end{tabular}

